\title{
Transoral decompression and posterior stabilisation in Morquio's disease
}

\author{
J Ashraf, H Alan Crockard, Andrew O Ransford, John M Stevens
}

\begin{abstract}
A 3.5 year old boy with Morquio's disease was referred with a persisting left hemiparesis four months after a fall and was found to have craniocervical junction compression due to atlantoaxial subluxation and significant anterior soft tissue compression. Transient unconsciousness at the time of the fall was probably due to medullary concussion as a result of hyperextension, not a head injury.

Spinal cord compression due to atlantoaxial subluxation at the craniovertebral junction is a major cause of disability and death in these patients. Once cervical myelopathy appears, early posterior occipitocervical fusion has been advocated in order to arrest the progression of neurological disability and this is successful in most cases. This conventional approach was considered unsafe because of the significant anterior compression. A combined anterior transoral decompression with posterior fusion to deal with this particularly difficult problem is described.
\end{abstract}

Morquio's disease (mucopolysaccharidosis type IV) is an inherited connective tissue disorder caused by absence or reduction in activity usually of one of two ubiquitous lysosomal hydrolases, $\mathrm{N}$-acetylgalactosamine 6-sulphatase (MPS IV type A) and $\beta$-galactosidase (MPS IV type B). ${ }^{1}$ It manifests clinically as a disproportionate dwarfing dysplasia with hypermobility of the joints due to laxity of restraining ligments and numerous other features. Cardiac defects and spinal cord compression are major causes of disability and death. Spinal cord compression in the thoracolumbar and sometimes also in cervicothoracic regions is due to kyphosis and narrowing of the spinal canal and is usually less severe there than at the craniovertebral junction where localised soft tissue thickening is the dominant compressing agent. ${ }^{2}$ Prompt posterior occipitocervical fusion is advocated once clinical myelopathy is present ${ }^{3}$ and this procedure may result in regression of thickened soft tissue. ${ }^{2}$ However, most authorities agree that established neurological deficits usually do not improve, and operative mortality can be high. ${ }^{34}$

Modern computed imaging (computed tomography and magnetic resonance imaging) has emphasised that in some patients there is so little available space for the spinal cord, that posterior fusion may be hazardous; this suggested the logic of performing a prior anterior decompression. Moreover, immediate decompression of the spinal cord by this procedure might allow neurological recovery to take place. The authors describe a case managed in this way.

\section{Case report}

A boy was born after an uneventful pregnancy and delivery. There was no known consanguinity and his parents and other siblings were normal.

At birth he was noted to have a pigeon chest deformity, and during his early development he was of short stature and physically weak. He did not have any of the ocular or cardiac manifestations of Morquio's disease. Although his early developmental milestones such as sitting, smiling, and talking were normal and comparable with those of his siblings, he did not start to walk until the age of $2 \cdot 3$ years. Shortly after his third birthday he had a fall, and although he did not hit his head he was unconscious for a short period. On regaining consciousness, he was intellectually intact but had a left hemiplegia and was unable to walk. Plain radiographs revealed atlantoaxial subluxation, and he was placed in a soft collar. There was a gradual improvement in function but he had a residual left hemiparesis. He was able to sit, crawl, and grasp things with his left hand but was unable to stand or walk, and so, four months after the injury at 3.5 years, he was referred for consideration of decompression and stabilisation of his craniovertebral junction.

On examination he was short statured, with a prominent cranium and vault, pigeon chest deformity, and kyphoscoliosis. He had spatulate fingers and hypermobile joints. His mental development was normal for his age. His cranial nerves were normal. He was unable to stand and tended not to move his left arm, holding it in flexion most of the time. He had a left hemiparesis, but power was normal on the right. Tone was increased in the left arm and leg. Reflexes were generally brisk, more so on the left, with extensor plantar response on that side. There was no detectable sensory deficit and autonomic function was normal.

His corneas were clear, there was no evidence of cardiomegaly or cardiac valvular dysfunction, and abdominal examination was normal.

Computed tomographic myelography demonstrated appreciable compression of the spinal cord by thickened soft tissues in the region of the partly ossified odontoid, associated with atlantoaxial subluxation (figs 1 and 2) which reduced in extension, although this did not completely relieve the compression (figs 3 and 4).

Skull halo traction was used to give preliminary reduction then transoral excision of the

Correspondence to: $\mathrm{Mr}$ Crockard. 

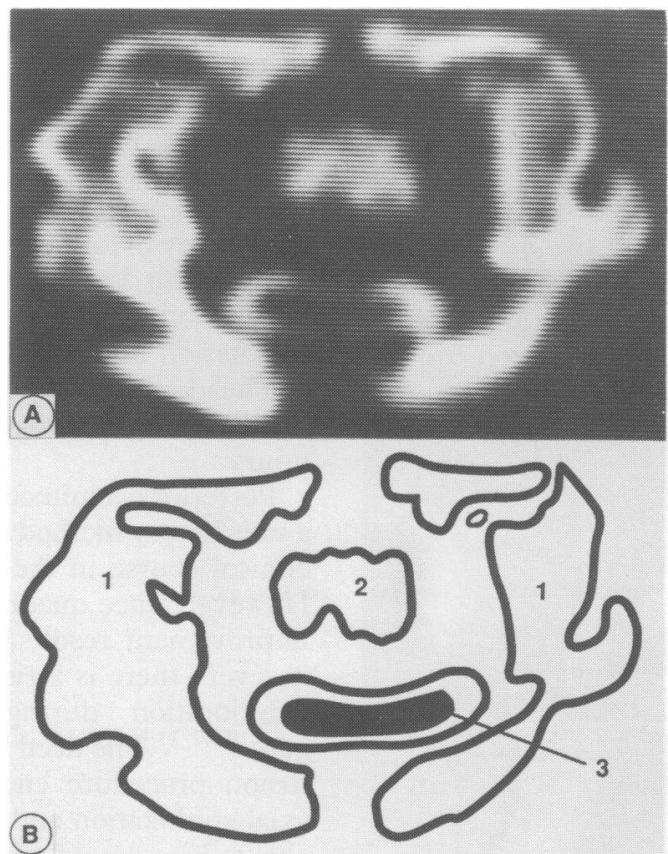

Figure 1 Axial computed tomographic myelogram $(A)$ and line diagram $(B)$ through the craniovertebral junction, showing compression of the spinal cord in flexion by the soft tissue surrounding a malformed odontoid process and anterior arch of atlas. $1=$ lateral mass of $C 1,2=$ odontoid base, $3=$ spinal cord odontoid, the body of $\mathrm{C} 2$ and the adjacent soft tissue elements compressing the spinal cord was performed, followed by a posterior occipitocervical fusion with a bone graft obtained from femur and tibia.

His recovery was uneventful. The halo body jacket was removed after six weeks, and his left hemiparesis improved considerably.

The histopathological examination of the excised tissue showed it to be composed of reactive tissue and partially ossified bone, with no evidence of abnormal mucopolysaccharide deposition.

\section{Discussion}

Abnormal development at the craniovertebral junction is an important feature of Morquio's disease. Plain radiographs of this region are often difficult to interpret ${ }^{5}$ and the following synopsis is based on more reliable observations made by computed myelography. ${ }^{26}$ In Morquio's disease, the odontoid process is detached from its basal portion and ossifies late, the atlas ring is small with an abnormally thick neural arch, and midline fusion defects are usually present. Although both anterior and posterior atlantoaxial subluxation are demonstrable, they seldom are severe and the bony spinal canal
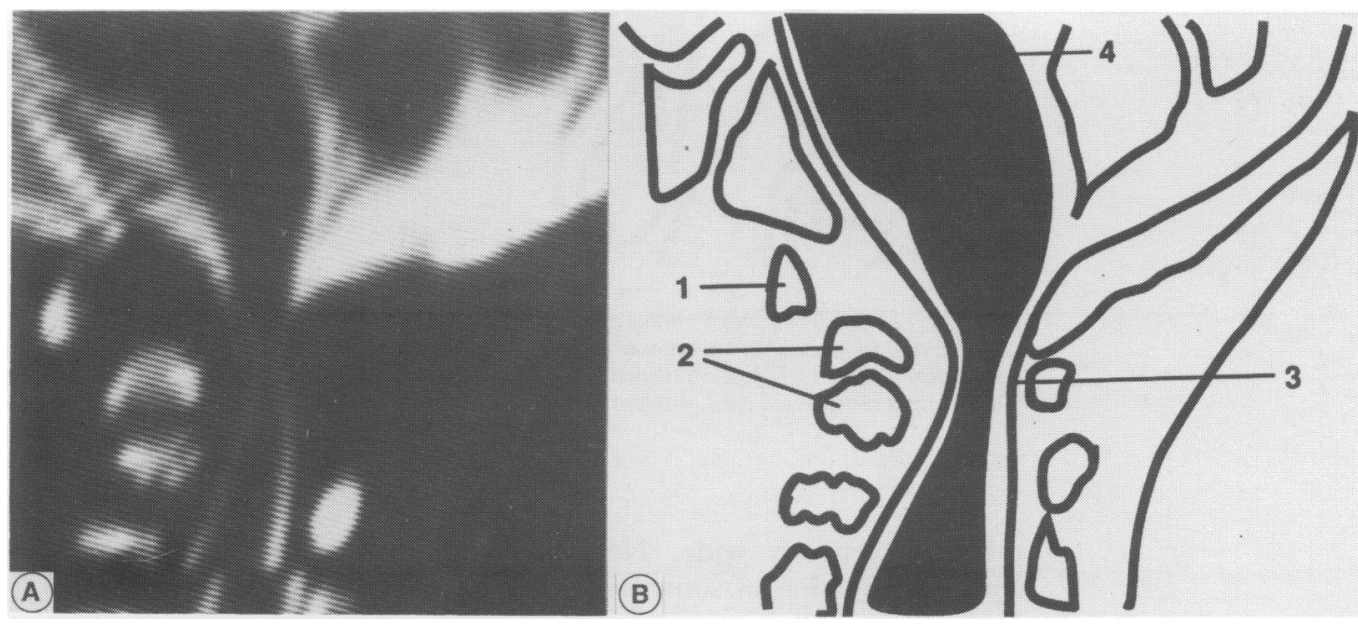

Figure 2 Sagittal computed tomographic myelogram $(A)$ and line diagram $(B)$ showing atlantoaxial subluxation, hypoplastic odontoid process, and soft tissue elements compressing the spinal cord. $1=C 1,2=$ odontoid base and body of $C 2,3=$ cerebrospinal fluid around compressed cord, $4=$ fourth ventricle.
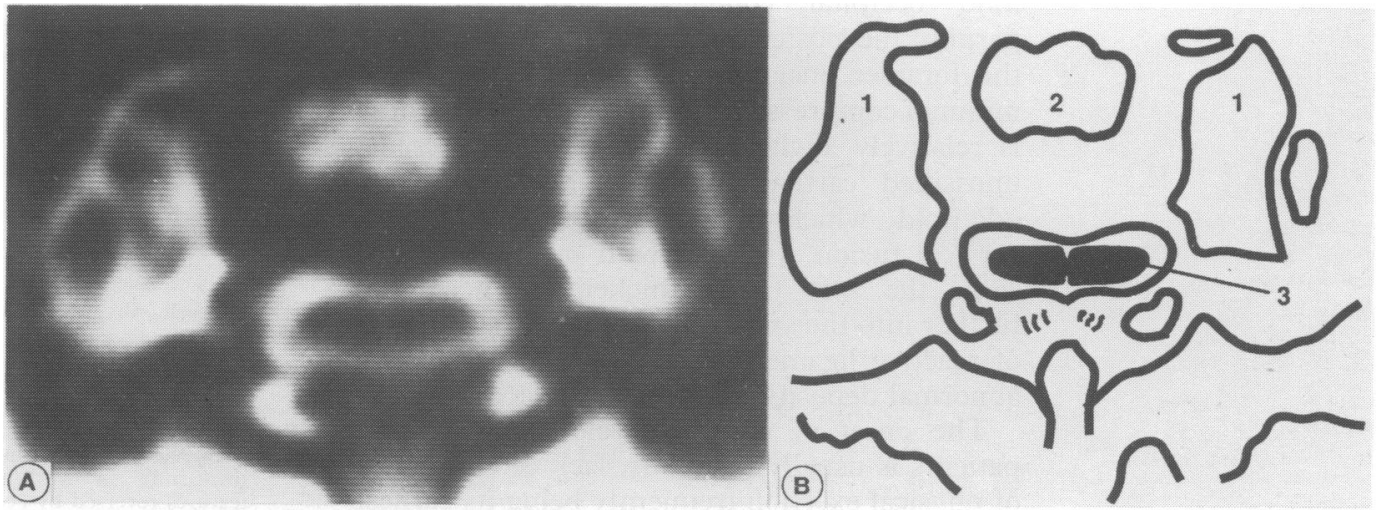

Figure 3 Axial scan $(A)$ and line diagram $(B)$ of the same area in extension, showing that the compression is reduced. $1=$ lateral mass of $C 1,2=$ odontoid base, $3=$ spinal cord. 


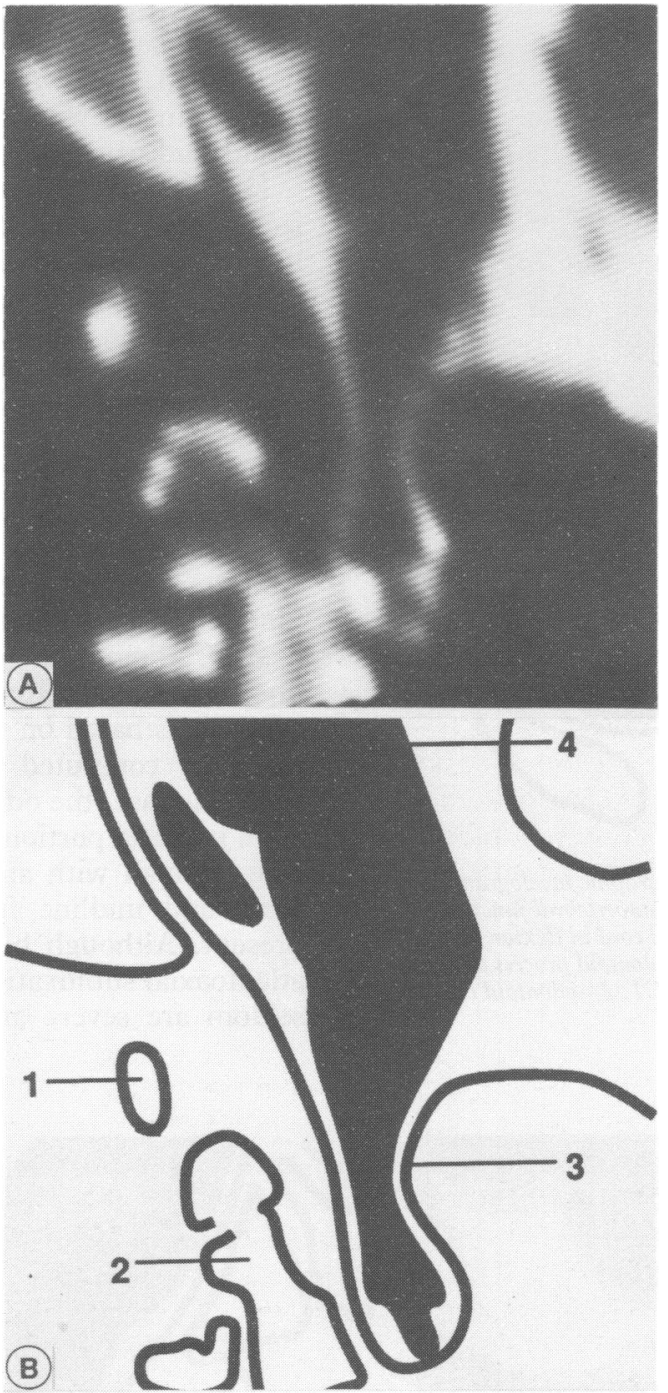

Figure 4 Sagittal computed tomographic myelogram $(A)$ and line diagram $(B)$ showing in extension the persisting cord deformation. $1=C 1,2=a x i s$ body, $3=$ cerebrospinal fluid around compressed cord, $4=$ fourth ventricle.

remains relatively wide. Nevertheless, spinal cord compression of some degree is present in most cases, and is severe in about $50 \%$. The cause is narrowing of the available subarachnoid space mainly by thickening of the anterior extradural soft tissues, especially in the region of the odontoid, accentuated in some cases by mild occipital condylar hypoplasia which permits the posterior arch of $\mathrm{Cl}$ to invaginate the foramen magnum. In cases with mild or minimal compression, the soft tissue thickening is relatively slight; in the main it represents unossified cartilage in vertebral bodies and odontoid, which usually ossify after occipitocervical fusion. In cases with severe compression, the soft tissue thickening encroaches further into the spinal canal and consists in part of reactive ligamentous tissue with or without abnormal deposition of mucopolysaccharide.

The onset of clinical myelopathy in these patients is usually insidious, lack of endurance of physical exertion frequently being its earliest manifestation. ${ }^{3} 7$ Subsequent progression is variable, often with periods of sudden severe functional loss which cannot always be related to trauma. Generally, progression is slower in older patients, ${ }^{389}$ often remaining undetected for long periods, and is more difficult to arrest or reverse by operation. A sudden hyperextension movement, as in a fall, may cause spinal cord injury of ranging severity or even medullary dysfunction leading to respiratory arrest. We believe our patient was unconscious due to concussion of the medulla rather than because of a head injury. Motor deficit without a sensory loss is also well recognised in upper spinal cord injury.

Posterior occipitocervical fusion appears to be a satisfactory method of arresting the downward clinical course in the majority of cases. ${ }^{341011}$ However, once quadriplegia is established little improvement results, ${ }^{8}$ and where compression is severe there is a risk of precipitating sudden deterioration during anaesthesia and surgery. ${ }^{912} 13$ The need to flex the neck during the fusion procedure encourages anterior atlantoaxial subluxation and further reduces the available subarachnoid space.

There are three advantages of an initial transoral exision of the anterior extradural soft tissue elements in patients with severe compression. (1) The operation is performed with the head extended, a position in which any anterior atlantoaxial subluxation is reduced and usually the available subarachnoid space maximised. (2) Adequate decompression permits safer posterior fusion. (3) Excision of the anterior soft tissue mass often is the only way of achieving immediate decompression of the spinal cord, thereby increasing the chances that even an established deficit might improve. We believe all three advantages were demonstrated by our patient. While it is conceded that transoral surgery adds significant surgical trauma to an already extensive procedure, in practised hands it extends the operation time by little more than an hour. In a wide range of pathology, intraoral excisions have healed rapidly with low risk of infection and morbidity. ${ }^{14} 15$

In conclusion, patients with Morquio's disease and mild spinal cord compression requiring operation are probably best treated by a posterior occipitocervical fusion. Patients with severe spinal cord compression, on the other hand, may require anterior decompression before safe stabilisation can be performed by the posterior approach, and this also may offer a better chance of reversing the established neurological deficit.

The authors are grateful to Michelle Green for her secretarial services. This work has been supported in part by the generous donation of Lex Brookland.

1 McKusick VA, Neufeld EF. The mucopolysaccharide storage diseases. In: Stanbury JB, Wyngarden JB. Fredrickson DS, Goldstein JL, Brown MS, eds. The metabolic basis of inherited diseases. 5th ed. New York: metabolic basis of inherited

2 Stevens JM, Kendall BE, Crockard HA, Ransford AO. Maldevelopment of the odontoid process and soft tissue thickening in Morquio-Brailsford disease: consisten features reversed by occipito-cervical fusion. 7 Bone foin Surg [Br] 1991;73:851-8.

3 Kopits SE. Orthopedic complications of dwarfism. Clin Orthop 1976;114:153-79. 
4 Greenberg AD. Atlanto-axial dislocations. Brain 1968;91: 655-84.

5 Ogden JA, Murphy MJ, Southwick WO, Ogden DA. Radiology of post-natal skeletal development XIII. Cl-C2 interrelationships. Skeletal Radiol 1986;15:433-8.

6 Edwards MK, Harwood-Nash DC, Fitz CR, Chuang SH. CT metrizamide myelography of the cervical spine in Morquio's syndrome. AFNR 1982;3:666-9.

7 Kopits SE, Perovic MN, McKusick V, Robinson RA, Bailey $\mathrm{JA}$. Congenital atlanto-axial dislocations in various forms of dwarfism. F Bone foint Surg [Am] 1972;54-A:1349-50.

8 Lipson SJL. Dysplasia of the odontoid process in Morquio's syndrome causing quadriparesis. $\mathcal{F}$ Bone foint Surg [Am] syndrome causing

9 McKusick VA. Heritable disorders of connective tissue, 4th Ed. St Louis: CV Mosby, 1972:596.
10 Bethem D, Winter RB, Luther L, et al. Spinal disorders of dwarfism: review of the literature and report of eighty cases. F Bone foint Surg [Am] 1981:63-A:1412-25.

11 Goldberg MJ. Orthopedic aspects of bone dysplasia. Orthop Clin North Am 1976:7:445-6.

12 Beighton P, Craig J. Atlanto-axial subluxation in the Morquio syndrome: report of a case. $\mathcal{F}$ Bone foint Surg [Br] 1973;55B:478-81.

13 Jones AEP, Croley TF. Morquio's syndrome and anaesthesia. Anaesthesiology 1979;51:261-2.

14 Crockard HA. Anterior approaches to lesions of the upper cervical spine. Clin Neurosurg 1988;34:389-416.

15 Crockard HA, Calder I, Ransford AO. One stage transoral decompression and posterior fixation in rheumatoid atlanto-axial subluxation. F Bone foint Surg [Br] 1990;72B:682-5.

Oculocerebrorenal syndrome of Lowe

This $\mathrm{X}$ linked recessive disorder has been mapped to the long arm of the X chromosome (Xq 24-26). Its three cardinal features are congenital cataracts, cognitive impairment, and renal tubular dysfunction. Other clinical features include hypotonia, glaucoma, and joint swellings. Eighty two patients with this syndrome are known to the American Lowe's Syndrome Association and 15 of those together with an additional eight are the subjects of a recently published study from the National Institutes of Health in Bethesda, Maryland (Lawrence R Charnas and colleagues, New England Fournal of Medicine 1991;324:1318-25).

Nineteen of the 23 patients were aged less than 17 years, the youngest being 4 months old. The other four were over 22 years old, the oldest being 31 .

Weight and length were normal at birth and in infancy but growth slowed from the age of 2 or 3 years, weight following a curve below but parallel to the third centile and height following a line which fell progressively away from the third centile. Growth often continued well beyond the normal age but the mean final height of the four adult patients was below the third centile. Head circumferences were within the normal range. With increasing age bone age lagged progressively further behind chronological age and height age behind bone age so that bone age was between chronological age and height age.

Serum creatinine appeared to increase stepwise with age, increasing fairly dramatically in patients over the age of 15 years. In 10 patients aged under 10 years the serum creatinine ranged from 27 to $53 \mu \mathrm{mol} / \mathrm{l}$. Between 10 and 15 years the range was 62 to 80 and after 15 years, 115 to $292 \mu \mathrm{mol} / 1$. The authors suggest that renal failure in Lowe's syndrome will occur at around the age of 36 years but that seems a fairly tenuous suggestion from the data presented.

Urine osmolality was low in 21 of the 23 patients and all but one had proteinuria. In 15 patients urine protein excretion was in the nephrotic range $\left(>1.0 \mathrm{~g} / \mathrm{m}^{2} /\right.$ day $)$ but they had no other features of the nephrotic syndrome. Renal tubular wastage of potassium, phosphate, and carnitine were common. Supplements of potassium were needed by eight patients, phosphate by seven, and two received L-carnitine. Fifteen patients took oral bicarbonate or citrate. Significant glycosuria was uncommon, only two patients showing a trace of glucose on dipstick testing. The amino aciduria of Lowe's syndrome seems to be less than is seen in cystinosis with little excretion of the branched chain amino acids, leucine, isoleucine, and valine. Raised serum enzyme activities including creatine kinase lead to the suggestion that there may be mild muscle disease in this syndrome contributing to the hypotonia.

Lowe's syndrome is rare but this paper gives valuable information about prognosis and about the findings to be expècted at various ages. 